\title{
The city [within] the drawing: The urban in the space of representation
}

\section{Sophia Banou}

The City [within] The Drawing is the last of a series of four installations made in the course of a $\mathrm{PhD}$ by creative practice. ${ }^{1}$ These installations posed questions about the material and temporal limits of conventional codes of architectural drawing and together attempted to forge an alternative urban representation. The series concluded in The City [within] The Drawing as a cumulated transcription within which the previous installations were embedded. This last installation expressed the negotiations that emerge between the actuality of the city and its image, by engaging with architectural representation $^{2}$ as an act of inhabitation ${ }^{3}$ performed through the spatial experience of both city and drawing.

As an attempt to 'draw' the actual city into representation, mapping was deployed as a kind of inhabitation that promotes a reciprocal close 'looking' between the city and the drawing.
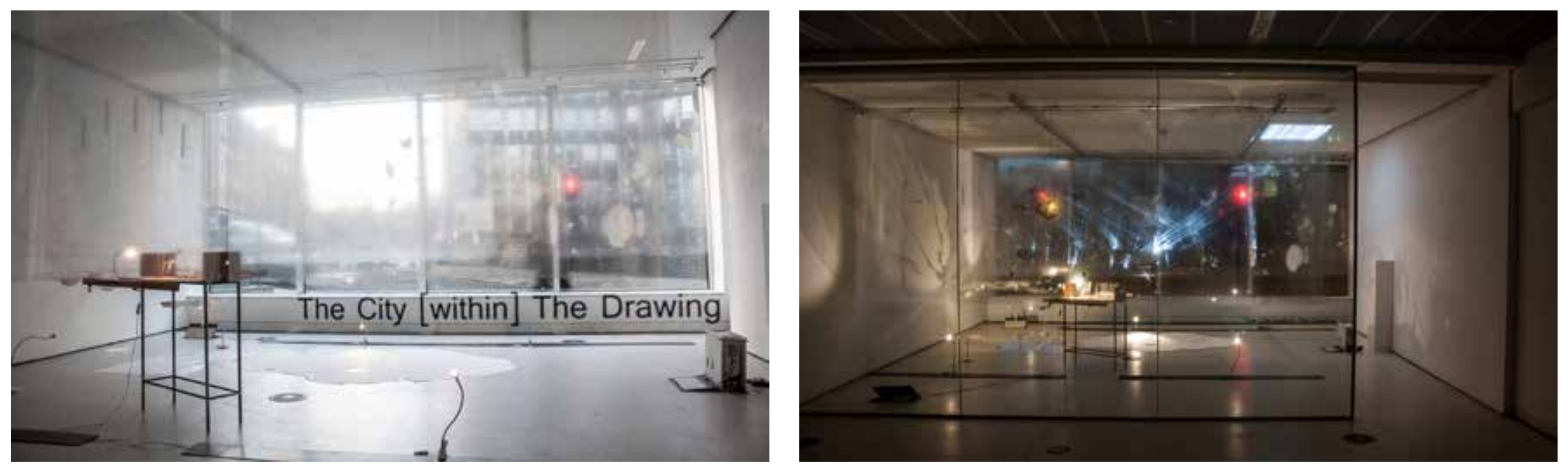

Figs. $01 \& 02$

Sophia Banou (2015). The City [within] The Drawing: General views across the

installation and to the city [Installation, Photos: Author] 
Inhabitation, therefore involves engaging with the 'material' trace of not only the city, but also the observer and the notation itself. Shifting focus from a normative architectural solid-void dualism, the project addressed the urban as a transitory condition between order and event. Inscribed elements printed across suspended layers of clear acetate presented a constellation of marks that corresponded to specific trajectories, relationships of vision, and transformations of the materiality of both the city and the (preceding) installations. These markings grafted conventional codes from dance notation, geological mapping, and celestial cartography into architectural convention.

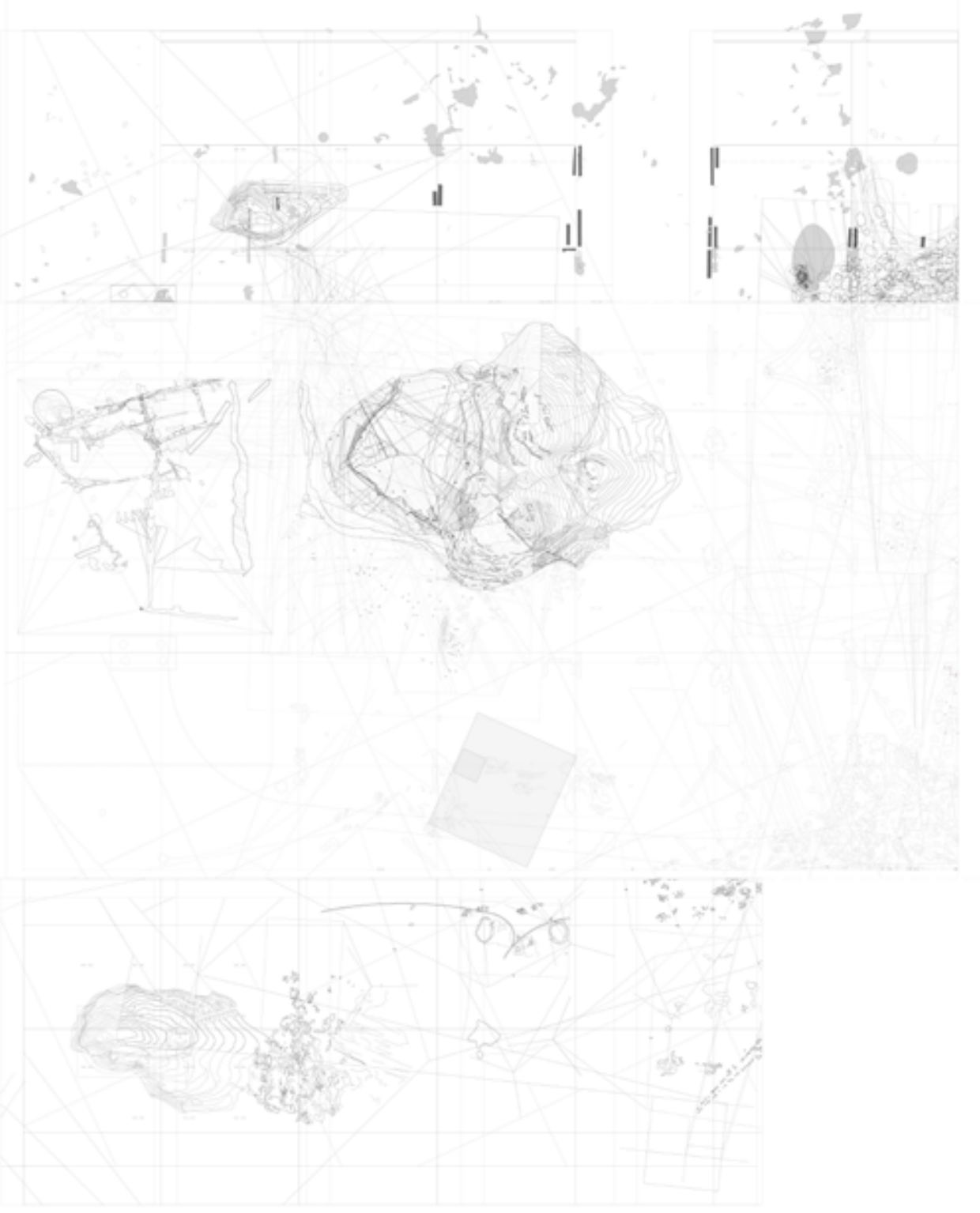

Fig. 03

Sophia Banou (2015). The City [within] The Drawing: The total of traces unfolded against a spread of the gallery [Installation, Photos: Author] 


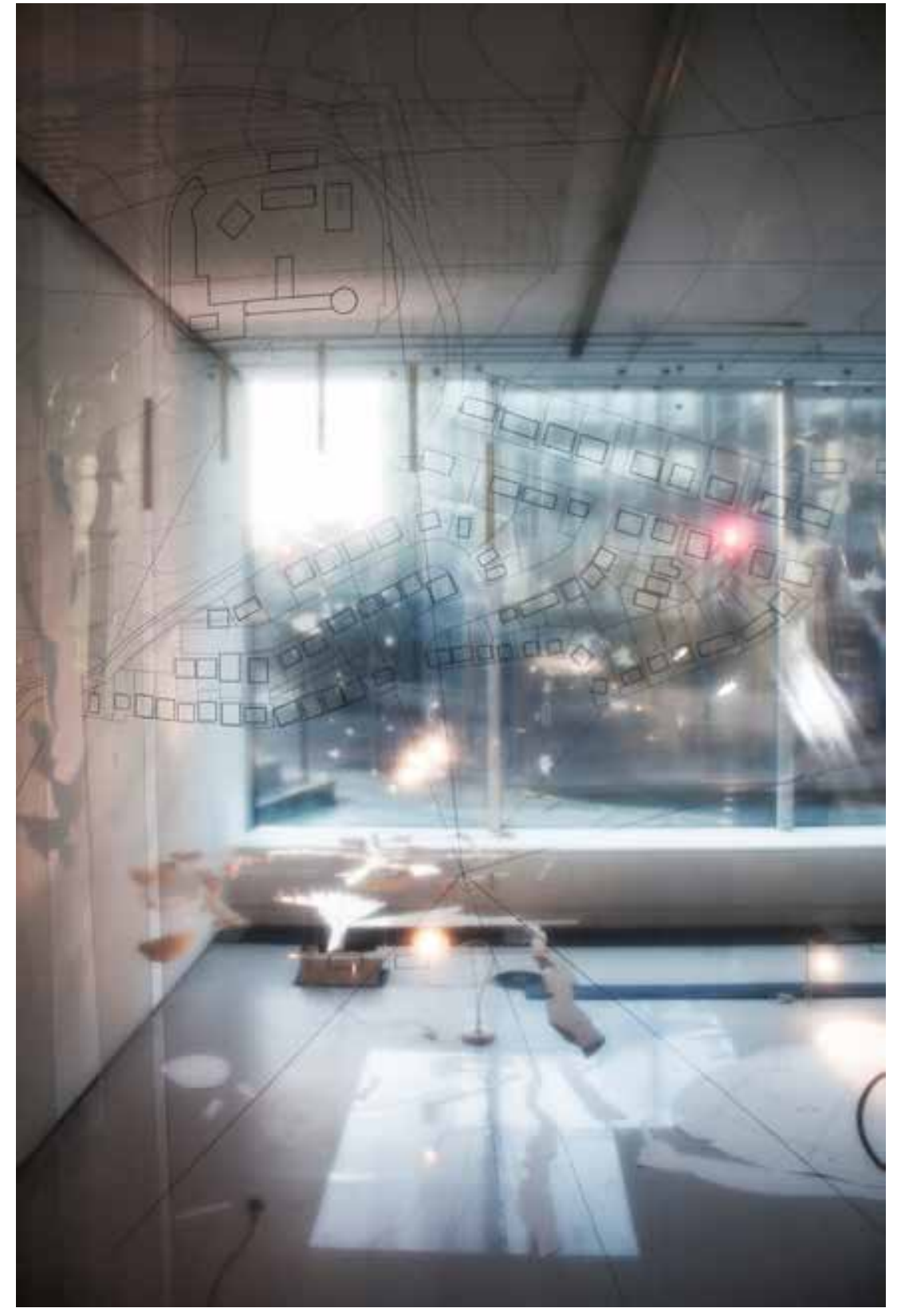

Provisionally reterritorialized upon a transparent substrate material, these signs were constantly repositioned against the city and the space of the gallery through the relational movement of the viewers.

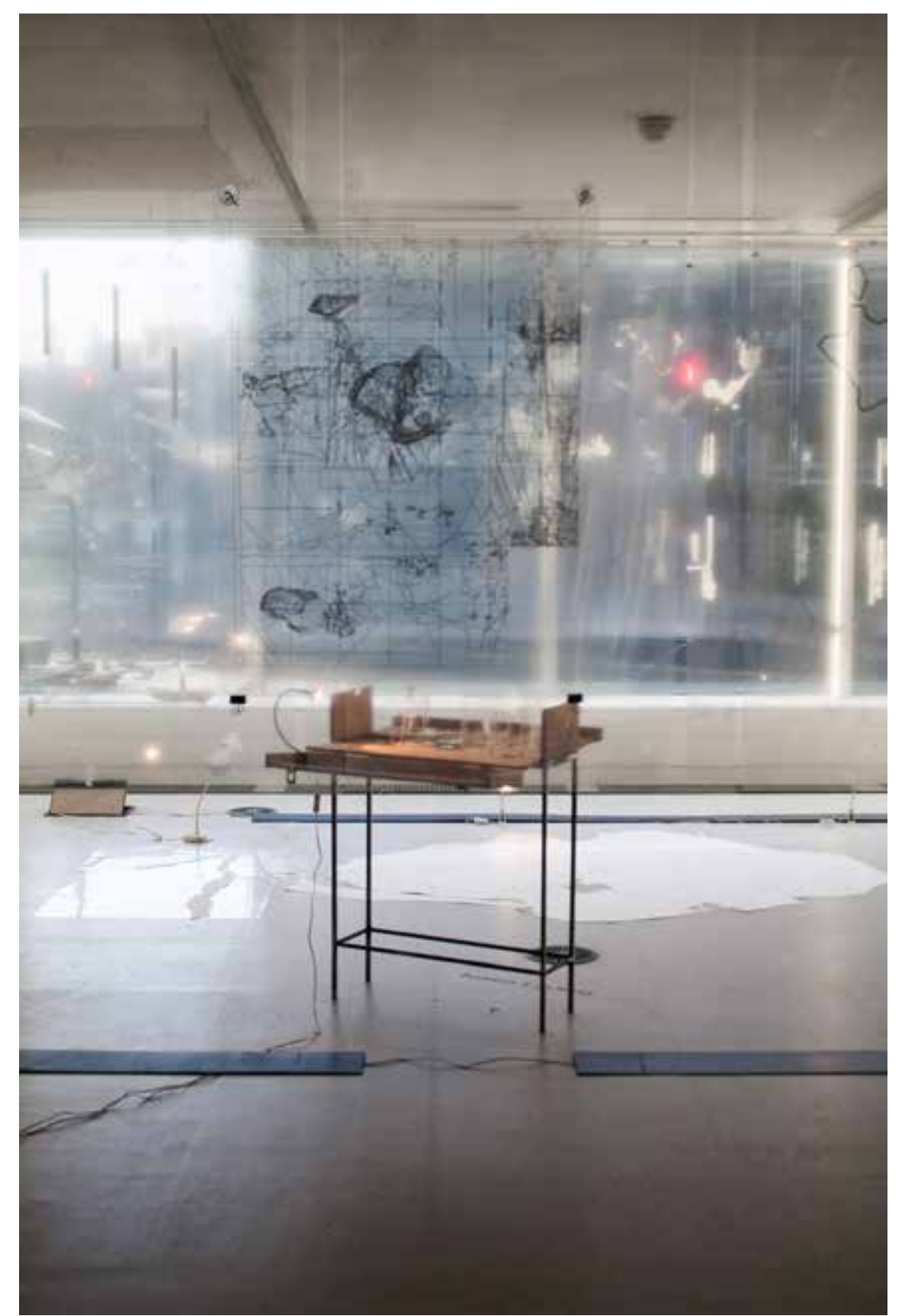

Figs. $04 \& 05$

Sophia Banou (2015). The City [within] The Drawing: Planar traces on the suspended clear acetate prints [Installation, Photos: Author]

The nesting of the drawing's earlier phases within one another was extended to the city through the inclusion of the direct city views from the gallery that layered with the transcribed traces. 

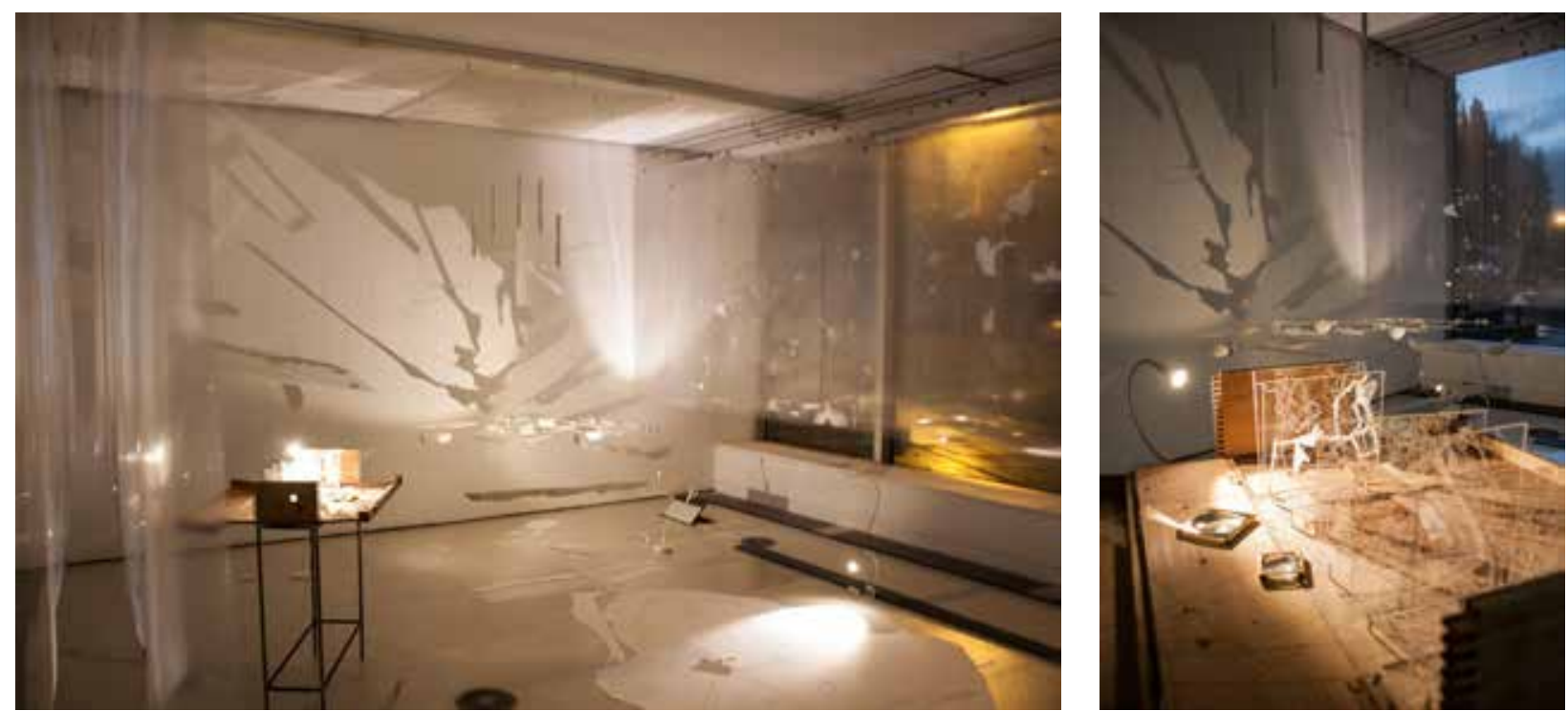

Figs. 06 \& 07

Sophia Banou (2014). Draw of a Drawing: One of the preceding representations, 'nested' in The City [within] The Drawing, stood as a partial 'key' to the installation [Portable installation, Photos: Author]"]

The multiple layers of the drawing carried the presence of the previous installations, not as mere signs but as distinct situated spatialities. Elements of previous sitings such as markings and scars were stenciled on polyester sheets to infuse the transparent layers with the neutral 'white' of the gallery. The solidity of the gallery floor afforded the signs of the various layers to become fixed upon the gallery surface,
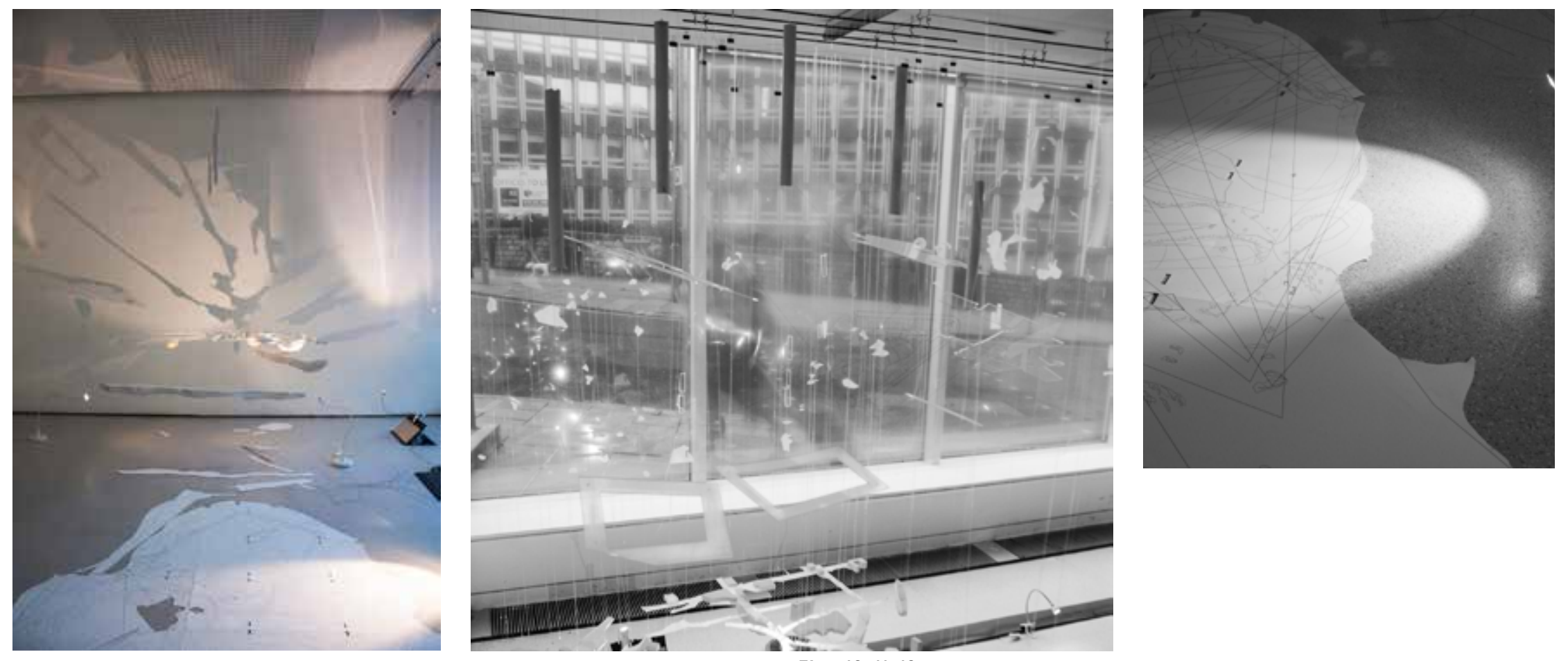

Figs. 10, 11, 12

Sophia Banou (2015). The City [within] The Drawing: The City Model, where surface elements are materialised in plaster and visual relations are concretised in sanded acrylic, emerging from floor [Installation, Photos: Author]

whilst modelled elements sought to 'push through' the two-dimensionality of the drawing surface. This served to materialise and project back the 'city' emerging from within the drawing, as at once 'other' to and the same as its onlooker. 

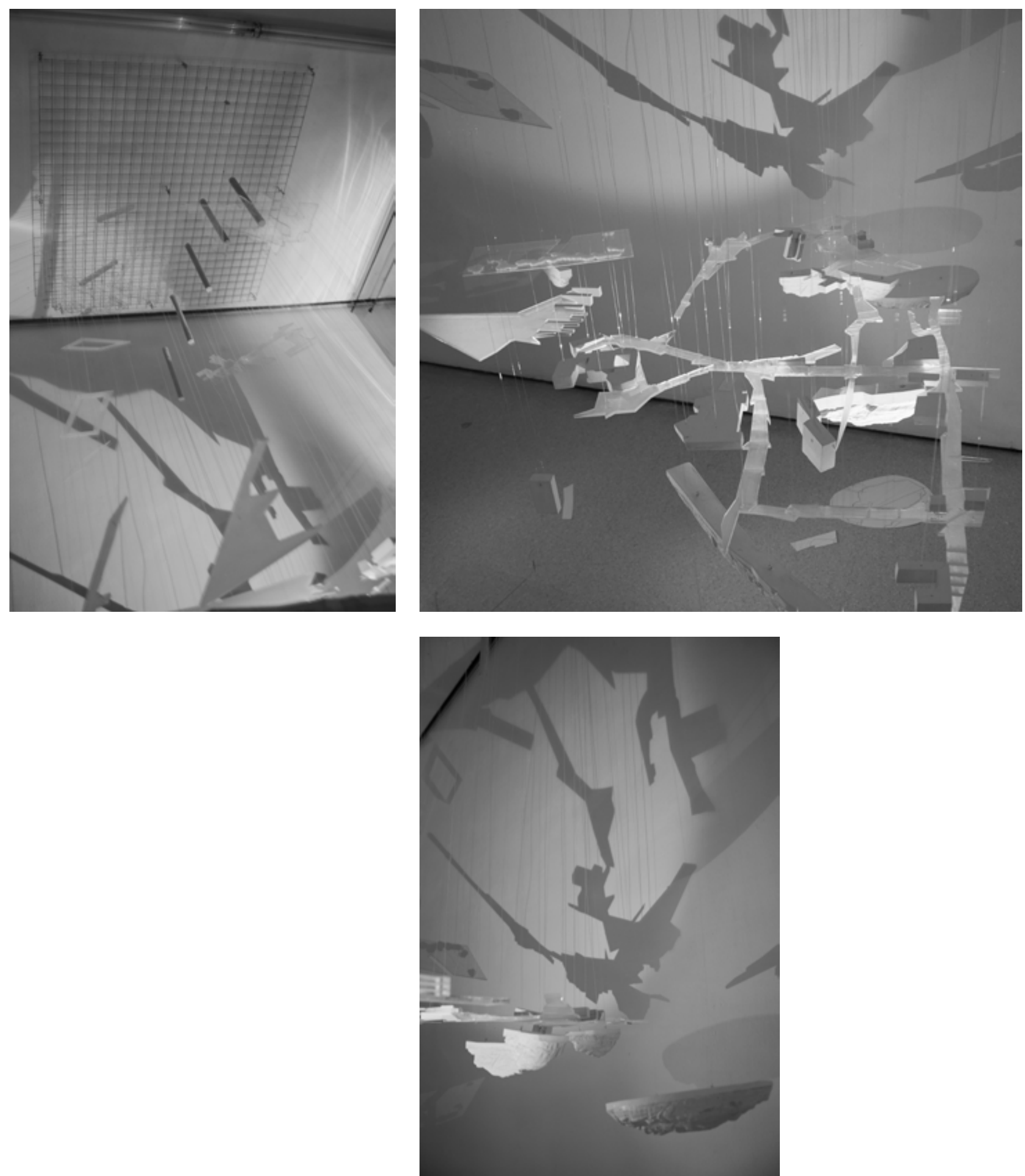

Figs. 13, 14, 15

Sophia Banou (2015). The City [within] The Drawing: Views of the City Model [Installation, Photos: Author]

The installation, as a situated experience of drawing, performed as a 'mediating object' between city and spectator, prompting conditions of observation to persistently reconfigure by placing the two in mutually shifting relations. 

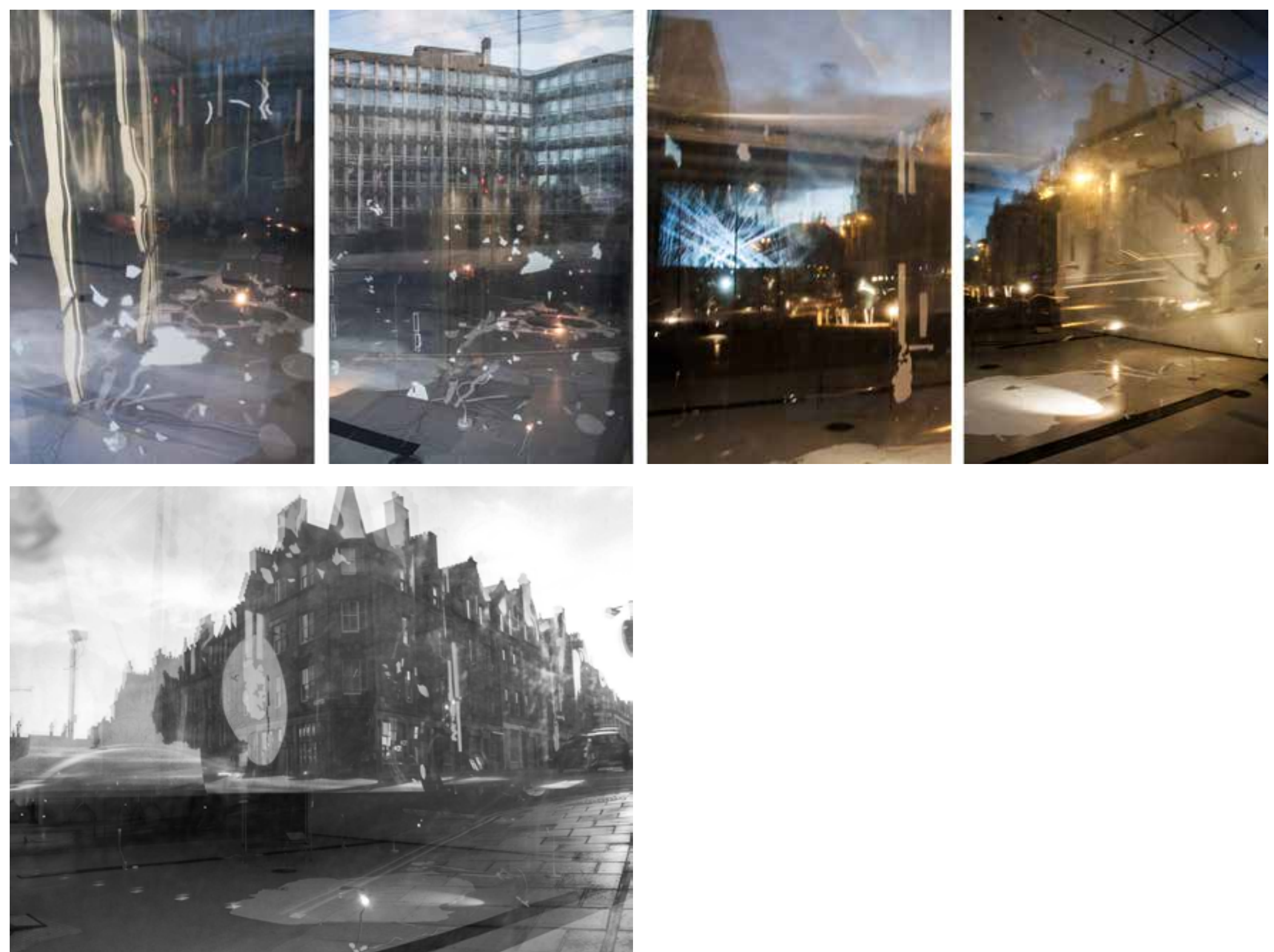

\section{Figs. 08 \& 09}

Sophia Banou (2015). The City [within] The Drawing: Views from the city into the drawing/installation [Installation, Photos: Author]

In this recursive process between sites of origin and sites of representation, the medium of installation posed as an opportunity to be immersed in, or rather inhabit, a 'space of representation', as the material recoding of the city within an expanded field. This representational field incorporated the kind of objects and conditions upon whose exclusion the coherence of the city had previously depended. 


\section{Endnotes}

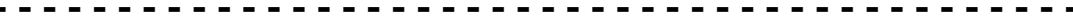

1 The installation was presented in March 2015, at the Tent Gallery, Edinburgh College of Art, from research undertaken at the University of Edinburgh, supervised by Prof Mark Dorrian and Dr Ella Chmielewska. On the preceding installations see Banou, S. (2015). Animated Gazes: Motion and Representation in the Kaleidoscopic City. Drawing On, 01: Presents. Retrieved from http://www.drawingon.org/issue/01, and Banou, S. (2015). Deep Surface: On the Situation of Drawing. Inflection 02: Projection, 76-83.

2 The object of this representation was the city of Edinburgh, however and despite the operative character of the site-specificities that emerged, the questions raised and the effects produced should not be considered as pertaining to this particular city, as they have been driven by a concern with the agency of drawing rather than the specific characteristics of the city represented.

3 The idea of drawing as inhabitation is based on its understanding as a site-specific act and can be traced to notions of subjectification that emerged in cartographic approaches to architectural representation and specifically the understanding of representation as site in the work of Metis. See Heidegger (1993) Dorrian \& Hawker (2002). 
References

Heidegger, M. (1993). ‘Building, Dwelling, Thinking’. In Farrell Krell, D. (Ed.), Basic Writings (pp. 356-). London: Routledge.

Dorrian, M. \& Hawker, A. (2002). 'Postscript as Pretext’. In Metis: Urban Cartographies. London: Black Dog Publishing. 